\title{
Colorectal Cribriform Comedo-Type Adenocarcinoma
}

National Cancer Institute

\section{Source}

National Cancer Institute. Colorectal Cribriform Comedo-Type Adenocarcinoma. NCI

Thesaurus. Code C96488.

A rare, invasive colorectal adenocarcinoma characterized by the presence of malignant cribriform glands with central necrotic changes. 